Estudios de Psicología, vol. 22, núm. 2, 2001, pp. 207-226.

\title{
Creación de símbolos y ficción durante el segundo año de vida.
}

\section{Español, Silvia.}

Cita:

Español, Silvia (2001). Creación de símbolos y ficción durante el segundo año de vida. Estudios de Psicología, 22 (2), 207-226.

Dirección estable: https://www.aacademica.org/silvia.espanol/118 ARK: https://n2t.net/ark:/13683/pH0V/cwS 
This article was downloaded by: [UAM University Autonoma de Madrid]

On: 20 J une 2014, At: 06:28

Publisher: Routledge

Informa Ltd Registered in England and Wales Registered Number:

1072954 Registered office: Mortimer House, 37-41 Mortimer Street, London W1T 3J H, UK

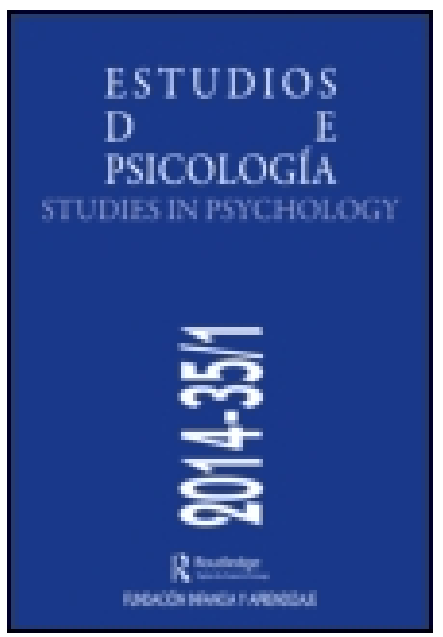

\title{
Estudios de Psicología: Studies in Psychology
}

Publication details, including instructions for authors and subscription information: http:/ / www. tandfonline.com/loi/ redp20

\section{Creación de símbolos y ficción durante el segundo año de vida}

\author{
Silvia Español ${ }^{a}$ \\ a Universidad de Buenos Aires \\ Published online: 23 J an 2014.
}

To cite this article: Silvia Español (2001) Creación de símbolos y ficción durante el segundo año de vida, Estudios de Psicología: Studies in Psychology, 22:2, 207-226, DOI: 10.1174/021093901609505

To link to this article: http:// dx. doi.org/ 10.1174/ 021093901609505

\section{PLEASE SCROLL DOWN FOR ARTICLE}

Taylor \& Francis makes every effort to ensure the accuracy of all the information (the "Content") contained in the publications on our platform. However, Taylor \& Francis, our agents, and our licensors make no representations or warranties whatsoever as to the accuracy, completeness, or suitability for any purpose of the Content. Any opinions and views expressed in this publication are the opinions and views of the authors, and are not the views of or endorsed by Taylor \& Francis. The accuracy of the Content should not be relied upon and should be independently verified with primary sources of information. Taylor and Francis shall not be liable for any losses, actions, claims, proceedings, demands, costs, expenses, damages, and other liabilities whatsoever or howsoever caused arising directly or indirectly in connection with, in relation to or arising out of the use of the Content.

This article may be used for research, teaching, and private study purposes. Any substantial or systematic reproduction, redistribution, 
reselling, loan, sub-licensing, systematic supply, or distribution in any form to anyone is expressly forbidden. Terms \& Conditions of access and use can be found at http://www.tandfonline.com/page/ terms-and-conditions 


\title{
Creación de símbolos y fícción durante el segundo año de vida
}

\author{
SILVIA ESPAÑOL \\ Universidad de Buenos Aires
}

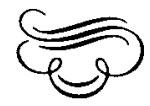

\begin{abstract}
Resumen
Resumen

A pesar de lo mucho que se ha escrito sobre la comunicación simbólica y la capacidad de ficción en el desarrollo ontogenético, son muchos aún los interrogantes sin respuesta. Ángel Rivière elaboró una concepción novedosa acerca del simbolo enactivo y la ficción, en el contexto amplio de su teoría de semiosis por suspensión, que responde a algunos de ellos. Especificamente, su teoría brinda una bipótesis original acerca de la naturaleza y la constitución de ambos. A la luz de sus ideas y sobre la base de un estudio longitudinal de dos casos únicos, en este trabajo se analizan los sucesivos pasos en el desarrollo que permiten la construcción de símbolos enactivos y la creación de ficción en el período evolutivo comprendido entre los doce y los veinticuatro meses.
\end{abstract}

Palabras clave: Comunicación preverbal, gestos, gestos simbólicos, simulación, ficción, semiosis.

\section{Creating symbols and fiction during the second year of life}

\begin{abstract}
In spite of the great amount of literature on symbolic communication and the capacity to create fiction in ontogenetic development, there are still many unanswered questions. Angel Rivière developed a novel conception concerning fiction and enactive symbols within the context of bis theory of "semiosis by suspension". This conception is put into practice as an explanatory device in a longitudinal case study of two children, spanning a period between 12 and 24 months of age. This research work undertakes a developmental analysis of the processes involved in creating fiction and in developing enactive symbols.
\end{abstract}

Keywords: Preverbal communication, gestures, symbolic gestures, simulation, fiction, semiotics.

Agradecimientos: Lo que aquí se presenta forma parte del trabajo de tesis doctoral que realicé bajo la dirección de Ángel Rivière, a él mi reconocimiento y agradecimiento.

Correspondencia de la autora: Programa de Estudios Cognitivos. Facultad de Psicología. Universidad de Buenos Aires. Independencia 3065. Piso $3^{\circ}$ Box 8 (CP 1225). Buenos Aires. Argentina. Tel-Fax: 054-1-49575886 ó 054-1-4706-1983.E-mail: silviaes@psi.uba.ar 


\section{8}

\section{INTRODUCCIÓN}

Presentaré aquí algunas observaciones de la actividad de dos niños - Habib y Silvita - en situaciones de interacción natural conmigo. El análisis y la interpretación que haré de ellas están inexorablemente unidos al modo de comprender el desarrollo normal de Ángel Rivière que se encuentra deletreado en su teoría de semiosis por suspensión. He observado a los niños desde que tenían nueve meses hasta los veinticuatro, sin embargo me centraré en la descripción de los cambios que ocurren en los últimos doce meses. No es que considere que no pueden rastrearse los orígenes de las capacidades simbólicas y ficcionales en los meses anteriores, de hecho comentaré brevemente la evolución de las cualidades de la interacción y de las capacidades comunicativas que los anteceden y los tornan posibles; pero creo que, alrededor de los doce meses, puede encontrarse el inicio de un proceso que nos lleva directamente a ambos y que hasta ahora no ha sido descrito de esta manera, al menos hasta donde tengo noticias.

Mi objetivo es mostrar el proceso a partir del cual los niños son capaces de crear símbolos y juego de ficción a través de acciones y gestos, cómo construyen de modo idiosincrático ciertos signos que, a diferencia de los signos lingüísticos básicamente arbitrarios, son formas híbridas que conjugan a la vez una naturaleza social y motivada. Ambas creaciones semióticas —el símbolo enactivo y el juego de ficción — han sido explicadas por Ángel Rivière en su teoría de semiosis por suspensión (Rivière, 1997, 1998a, 1998b, 1998c; Rivière y Sotillo, 1999; Rivière y Español, en prensa). En ella son tratadas como los productos de dos de los cuatro niveles propuestos en la teoría. No quiero repetir lo ya dicho por mi maestro, más aún cuando no creo que sea posible decirlo de un modo más adecuado; me ocuparé en cambio de mostrar el sendero evolutivo que, de acuerdo a las observaciones interpretadas a la luz de su teoría, torna posible el tránsito de un nivel a otro o de una forma semiótica a otra. Sin embargo, para ello he de referirme brevemente a la teoría de semiosis por suspensión; sin pretender brindar una síntesis o resumen de ella, cosa que he hecho en otra ocasión (Español, en prensa), comentaré aquí sólo aquello que resulta imprescindible para el desarrollo de este escrito.

La teoría sigue las vicisitudes en el desarrollo ontogenético de un mecanismo particular de creación semiótica: el mecanismo de suspensión. Un mecanismo aparentemente simple que, sin embargo, puede explicar formas de semiosis extremadamente diversas. Suspender es, lisa y llanamente, "dejar algo sin efecto”, hacer que algo deje de tener los efectos normales que tendría sobre el mundo; es hacer que algo deje de regir (Rivière y Español, en prensa). Pese a su simpleza, el mecanismo enhebra capacidades que son hitos en el desarrollo evolutivo: los primeros gestos deícticos, los símbolos enactivos, el juego de ficción, la comprensión metafórica. ¿Cómo es posible que un único mecanismo tan simple pueda aunar fenómenos tan diversos? La respuesta se encuentra en la variedad de aquello que es dejado en suspenso, o lo que es lo mismo, en la fuente de suspensión. A medida que el niño domina ciertas formas de relación con el mundo, éstas se tornan fuentes posibles de ser dejadas en suspenso dando lugar a diversas formas de significar. La suspensión de una acción directa, una acción instrumental, una representación primaria del mundo, o una representación simbólica dan lugar a los primeros gestos deícticos, los símbolos enactivos, el juego de ficción, y la comprensión metafórica, respectivamente. Estos modos de significar son en su origen modos de significar para otros, surgen en el seno de situaciones comunicativas y se orientan hacia la interpretación de otro, siendo factibles luego de orientarse hacia ese particular interpretante, el sí mismo, que se construye a lo 
largo del desarrollo evolutivo. En la teoría se plantea un momento previo a todos los niveles que, a diferencia de ellos, no se presenta como un modo de producción sígnica en el que se pueda observar la presencia de una intención de producir un significado. Me refiero a las expresiones emocionales que son consideradas en la teoría el nivel 0 de suspensión. Rivière sugiere que las expresiones emocionales son el modo más primitivo de producción sígnica en el que actúa el mecanismo de suspensión (Rivière y Español, en prensa). A través de ellas iniciaré un breve recorrido por los intercambios sígnicos que anteceden a la creación de los símbolos enactivos.

A la temprana edad de dos meses, el bebé puede ser influido por la variación en las expresiones visibles y audibles de su madre; pero no sólo el bebé se muestra sensible a las expresiones de la madre, sino que ella también se acomoda a las expresiones de su hijo; es decir, madre e hijo entran en una regulación mutua de movimientos expresivos que permiten las primeras expresiones rudimentarias de intersubjetividad primaria (Trevarthen, 1982). Entre la madre y el niño se establece una interacción coordinada y armónica, un intercambio de signos, que conducen a un compromiso psicológico o una participación emocional compartida; se establece entre el adulto y el niño una ligazón subjetiva con la vida subjetiva del otro (Hobson, 1993). Se constituye, así, una experiencia empática de conexión entre el adulto y su cría en la que el bebé empieza a construir una cierta noción del otro, aún vaga, casi indiscriminada (Rivière y Sotillo, 1999). Por otro lado, el interés que los objetos despiertan en el niño desde los primeros meses es una condición indispensable para que, alrededor de los nueve meses, el niño transite desde el modo de intersubjetividad primaria a lo que Trevarthen denomina intersubjetividad secundaria. Un modo de estar con el otro en el que no sólo se comparten estados emocionales sino el interés hacia los objetos y eventos del mundo. Sus primeras manifestaciones se encuentran en las tempranas pautas de atención conjunta, por ejemplo en la alternancia de la mirada entre el otro y el objeto de interés. Estas situaciones, a las que a veces se las ha denominado situación primordial de compartir, indican una capacidad extremadamente simple pero crucial para el desarrollo: ponen de manifiesto que el niño es capaz de tener relaciones con los otros acerca de las cosas. Persona-objeto-niño forman un triángulo de relación. En ese momento están sentadas las bases para que, vía el mecanismo de suspensión, se produzca el tránsito de la acción al gesto y surjan los primeros gestos deícticos. Las acciones del niño orientadas hacia a los objetos, al entrar en interacción y mezclarse con las situaciones de atención conjunta o alternancia de la mirada comienzan a conformar la topografía de las interacciones comunicativas.

Así, los intentos fallidos del niño por coger un objeto, al producir una reacción no del objeto sino del adulto que se lo acerca, inducen al niño a reorientar su acción al adulto. Lo que era una acción cerrada se abre a la interacción y a la interpretación del otro: el niño realiza sólo el inicio de su acción mientras oscila la mirada entre el adulto y el objeto. Al iniciar la acción suspendiendo su ejecución completa, la acción directa de coger un objeto se transforma en el gesto de "extenderse hacia" o reaching (Franco y Butterworth, 1996; Español y Rivière, 2000); gesto en que la palma de la mano se encuentra hacia abajo con los dedos ligeramente curvados. Mediante la suspensión de la acción directa de tirar, en combinación con la suspensión de la acción triádica de dar, se constituye un gesto que parece especialmente diseñado para facilitar la obtención del contacto ocular y la atención conjunta a un objeto próximo: el gesto de mostrar, aquél en el cual el niño pone bajo la mirada del otro un objeto que tiene en la mano y, mientras alterna la mirada entre el objeto y el adulto, lo mueve hacia uno y otro 
lado (Español, 2001a). Mediante la suspensión de la acción de tocar, en íntima vinculación con lo que hemos denominado acto espistémico (Español y Rivière, 2000), se constituye el gesto de señalar.

Los gestos descritos provienen de acciones directas diferentes, pero el modo de constitución de ellos es el mismo: el paso de la acción al gesto está mediatizado por la suspensión de alguna acción directa, se constituyen mediante la realización del inicio de una acción directa, dejando "en el aire" el resto. Ellos suelen emerger hacia el final del primer año de vida. A diferencia de las formas primarias de intersubjetividad que se desarrollan durante los primeros meses que no parecen implicar aún ninguna conciencia, ni siquiera implícita, del otro como intérprete; estas pautas de acciones intencionadamente comunicativas implican algún grado de conciencia de estar produciendo una actividad que es meramente sígnica y que, por tanto, no ejerce efectos materiales sobre el mundo sino que tiene consecuencias mentales en los compañeros de interacción. Rivière enfatiza que estos modos de comunicación intencionada humana implican una cierta forma primaria de conciencia semiótica, una cierta "conciencia del otro como intérprete" (Rivière y Español, en prensa).

En ellos se anticipan por lo menos dos de las funciones con que posteriormente será usado el lenguaje: la función imperativa y la declarativa. Los gestos deícticos se usan, a veces, para pedir cosas (protoimperativos) y otras simple y maravillosamente, declarando, "comentando" la experiencia de un modo preverbal (protodelarativos). Un amplio grupo de investigadores se han ocupado de las habilidades sociales que ellos suponen (Bates 1976, 1979; Gómez, Sarriá y Tamarit, 1993; Perucchini y Camaioni, 1993; Phillips, Gómez, Baron-Cohen, Laa y Rivière, 1995; Goodhart y Baron Cohen, 1998; Gómez y Nuñez, 1998; Español y Rivière, 2000). Existe cierto acuerdo en que las pautas protoimperativas muestran los intentos comunicativos por lograr cambios en el mundo a través de las personas; las pautas protodeclarativas, en cambio, parecen tener una intención más genuinamente social: la de buscar compartir la atención y el interés por los estados del mundo. Ellas suponen la capacidad de percibir al otro como sujeto, como un ser subjetivamente orientado al mundo con el cual es posible compartir experiencias (Bretherton, McNew, Beeghly-Smith, 1981; Rivière y Nuñez, 1996; Hobson ,1993; Phillips et al., 1995). Indican la capacidad de suscitar intencionadamente condiciones que permiten compartir estados mentales (Rivière y Español, en prensa).

Un hecho curioso es que casi en el mismo momento en que los niños son capaces de dirigir la atención del otro señalando un objeto, son capaces también de dirigir el gesto a sí mismos. El señalar para sí mismo — que es actualmente foco de numerosas investigaciones (Camaioni, Perucchini, Murator y Milone, 1997; Franco y Butterworth, 1996; Butterworth, 1998; Español y Rivière, 2000) _ parece tener, llamativamente, una naturaleza social; es decir, si bien los niños señalan para sí mismos en situaciones de soledad lo hacen más frecuentemente cuando están con otros (Delgado, Gómez y Sarriá, 1999; Español y Rivière, 2000). La naturaleza social del señalar para sí mismo puede interpretarse como un indicador indirecto de la naturaleza social del sí mismo, al menos en tanto interpretante, que supone la presencia de un gesto autodirigido.

Hemos llegado a los doce meses, el momento en el que, creo, pueden seguirse la huellas de acciones y gestos que llevan directamente a la creación de los primeros símbolos enactivos. Antes de iniciar su recorrido intentaré responder de modo conciso dos preguntas que iluminarán, espero, el camino que invito a recorrer: ¿qué son los símbolos enactivos?, ¿en qué se diferencian de otros gestos simbólicos? 
Los símbolos enactivos son el producto del segundo nivel de suspensión. Si Ángel Rivière se refiere a los gestos deícticos como "metonimias enactivas", los símbolos enactivos son frecuentemente denominados por él "metonimias instrumentales". Son instrumentales porque la fuente sobre la que opera la suspensión es la acción instrumental; son metonimias en tanto suponen la realización de una parte de la acción dejando en suspenso el resto. Pero entre uno y otro nivel de suspensión hay una diferencia esencial que radica en la naturaleza diversa de la acción que es fuente de suspensión: en el primer nivel el carácter continuo de la acción restringe el modo de operar del mecanismo permitiéndole únicamente realizar el inicio de la acción dejando "en el aire” su continuación. En el segundo nivel, la naturaleza discreta de la acción instrumental permite un modo nuevo y distinto de producción sígnica. Un modo que no se encuentra constreñido a la realización del inicio de una acción directa sino que, dado su carácter discreto, permite la libertad de seleccionar una de las partes de la acción.

Los gestos deícticos no son creaciones originales sino que son comunes a todos los niños (por lo menos de nuestra cultura), son el resultado de la operación del mecanismo de suspensión a lo largo del proceso ontogenético. Los símbolos enactivos, en cambio, son gestos idiosincráticos en los que el mecanismo de suspensión opera en el momento que el niño genera el símbolo. Nacen de su necesidad de comunicarse acerca de objetos que no se encuentran presentes en el entorno inmediato, y que no son por tanto posibles de ser referidos de una manera deíctica (Rivière, 1990; Rivière y Sotillo, 1999). Los símbolos enactivos hablan de un modo libre, no copiado, de producción de significado; abren las puertas a un modo original y novedoso de referir, de aludir, a objetos y eventos del mundo y presentan un enlace entre significado y significante que no es arbitrario sino motivado. Por eso Ángel Rivière (1990) dice que tienen "vocación de metáforas”. Se constituyen mediante la imitación y selección de una parte de una acción instrumental; como cuando un niño, luego de llamar la atención de su padre, sopla un mechero que se encuentra apagado, pidiéndole así que lo encienda (Rivière, 1984). Son acciones desgajadas de su funcionalidad de primer orden que adquieren una funcionalidad nueva: la de representar algo (Rivière, 1984, 1990). Ellos se diferencian de los signos lingüísticos (en los que el enlace entre significado y significante es arbitrario, o al menos lo es para el niño que los adquiere) y se diferencian también de los gestos simbólicos que se describen en la mayoría de los trabajos sobre comunicación preverbal. Los gestos simbólicos que se describen en estos trabajos se aprenden por imitación y son tan arbitrarios como lo son los signos lingüísticos (Bates, 1979; Iverson, Capirci y Caselli, 1994; Tomasello y Camaioni, 1997). La teoría de semiosis por suspensión no se ocupa de ellos sino que se concentra en los símbolos que son creaciones motivadas e idiosincráticas.

Los símbolos enactivos denotan una especial concepción de la función simbólica en la que se encuentran presentes tanto ideas piagetianas como vygotskianas; pero aunque incorpore a ambas, de ambas también se distancia. Con un espíritu piagetiano en la teoría de semiosis por suspensión se defiende la existencia de símbolos no lingüísticos, creados enactivamente, motivados, que presentan una peculiar marca de deformación. Pero si la asimilación deformante sella la condición del símbolo piagetiano de ser algo más que una imitación pura, en los símbolos descritos por Rivière es la suspensión la que sella su condición de ser algo más que pura imitación. Los símbolos enactivos son una clase especial de gestos simbólicos que se tornan evidentes al ubicar la acción del niño en el escenario de la interacción con los otros y al observar el desarrollo de la acción del niño con esos objetos culturales, los instrumentos, que los adultos le ofrecen desde 
momentos tempranos. La influencia del pensamiento vygotskiano es evidente, pero la teoría de semiosis por suspensión se distancia también de él al reconocer la presencia de símbolos no lingüísticos en un período que Vygotski considera presimbólico. En realidad la concepción de símbolo de Rivière puede entenderse como un punto óptimo de tensión entre las dos grandes tradiciones en psicología del desarrollo (Español, 2001b).

\section{HACIA LA CREACIÓN DE SÍMBOLOS ENACTIVOS}

Dije que mi interés en este trabajo consiste en mostrar cómo se produce el tránsito entre algunos niveles de la teoría de semiosis por suspensión. Me ocuparé primero del tránsito del nivel 1 (gestos deícticos) al nivel 2 (símbolos enactivos), y posteriormente del tránsito al nivel 3 (juego de ficción). Lo que presentaré es un análisis parcial centrado en los aspectos y cambios morfológicos, los aspectos funcionales estarán menos desarrollados y las habilidades mentalistas que ellos suponen serán apenas señaladas. Motiva este sesgo la falta de espacio y la creencia de que el análisis minucioso de la forma es una buena plataforma para la comprensión global del fenómeno.

\section{Juego funcional, gestos de reconocimiento de objetos y gestos permutados}

Si se toman en cuenta los rasgos esenciales del punto de llegada del primer tránsito —el símbolo enactivo — es simple saber a qué tenemos que prestar atención si queremos observar su génesis: concentrar nuestra mirada en el despliegue de las actividades del niño con los instrumentos parece ser la ruta adecuada. Alrededor de los doce meses, es decir en el mismo momento evolutivo en el que los niños usan gestos deícticos para comunicarse con los otros, aprenden en colaboración con los adultos a utilizar instrumentos simples relacionados con sus actividades básicas (comer con cuchara, beber de un vaso); es decir aprenden a utilizarlos funcionalmente o con fines prácticos. Pero además, casi al mismo tiempo, realizan con ellos juego funcional: los usan de modo descontextualizado, sin que su acción tenga los efectos que tendría de realizarse efectivamente. A veces, imitan acciones instrumentales que ven realizar a los adultos, aunque no comprendan aún su significado, o lo comprendan parcialmente. A esta edad también aparece otro modo o actividad del niño con los instrumentos, surge algo así como una explosión de imitación de acciones instrumentales descontextualizadas pero realizadas de modo rápido y abreviado. Por ejemplo:

Al 1; 0 (17) Habib (H.) coge su teléfono de juguete se lo lleva a su oreja y enseguida lo deja.

Al 1; 0 (24) Silvita (S.) coge el cepillo y se peina sin mirar a nadie; luego coloca una diadema de su hermana en su cabeza; la saca y la deja en el suelo. Rato más tarde está con su muñeco, lo suelta sobre la mesa, coge un lápiz que estaba ahí, traza unas pocas líneas sobre la mesa y coge de nuevo su muñeco.

Al 1; 2 (19) S. busca en la bolsa, saca el cepillo, se peina y lo suelta, coge una cartera, la coloca en el hombro y la deja.

Al $1 ; 2$ (27) H. coge una cuchara la lleva a su boca, me la da y se dirige hacia los cubos. Al rato traigo un trapo de los que se usan para limpiar; H. pasa el trapo sobre la mesa una vez y lo deja. 
Alrededor de los doce-catorce meses, tal y como señalaron Rodríguez y Moro (1999), los objetos se tornan signos de su uso; despiertan inmediatamente la acción instrumental a la que se asocian y los niños la llevan a cabo de un modo descontextualizado, breve y conciso que las destaca y las diferencia del juego funcional.

El modo frecuente y abreviado en que los niños realizan acciones como éstas induce a pensar en ellas como un acto de reconocimiento del objeto, algo así como si dijeran mediante sus acciones "sé cómo se usa esto". Bates (1979) y Masur (1990) se refieren a ellos como "gestos de reconocimiento de objetos"; y sugieren que, al menos en algunos casos, son usados con la misma función que el señalar para sí mismo ya que frecuentemente se presentan de modo solitario, sin que el niño dirija su mirada al adulto. Esta hipótesis de similitud funcional da pie a considerarlos como una clase particular de gestos autodirigidos. Y efectivamente ésa es esa la impresión que se tiene al verlos realizar estas acciones instrumentales descontextualizadas, abreviadas, frecuentes y en numerosas ocasiones sin evidencia de que se dirijan a los otros, aunque producidas mayormente en situaciones de interacción. Si el señalar para sí mismo puede considerarse como un acto de reconocimiento del objeto, entonces es posible pensar que éste se continúa en un modo distinto de reconocimiento. En este caso, no se trataría de un reconocimiento de objetos que se encuentran a cierta distancia del propio cuerpo, o de un reconocimiento de objetos del mundo llamativos por sus características básicas (de color, forma y movimiento), sino de un reconocimiento del uso convencional, del carácter instrumental del objeto. Ahora bien, el gesto de señalar para sí mismo, en tanto gesto deíctico, es un gesto presimbólico, ¿los gestos de reconocimiento de objetos lo son también, o se trata de gestos simbólicos?

En la literatura sobre comunicación gestual éstos son frecuentemente tratados como gestos simbólicos (Bates, 1979; Iverson et al., 1994). Bates se refiere a ellos como actividades cortas y tenues de "pretend" o de "fingir que". Es cierto que si al llevarse la cuchara vacía a la boca, del modo específico en que ocurre en estas situaciones, los niños estuvieran haciendo "como que" o fingiendo que comen, de algún modo la comida estaría representada por un elemento similar pero no igual a ella (el gesto realizado con la cuchara) o el acto de comer estaría representado por una parte abreviada del acto completo. Habría entonces un diferencia entre el significante y el significado que estaría indicando una relación de representación en el que el primero "estaría por" el segundo. Los gestos de reconocimiento podrían verse como el uso de significantes, claramente diferenciados de sus significados, para remitir a objetos ausentes; es decir, como una acción simbólica al menos tal y como ha sido definida frecuentemente en psicología del desarrollo (Piaget, 1946; Bates, 1979; Rivière, 1984, 1990). Pero cuando un niño se lleva una cuchara vacía a la boca podría tan sólo tratarse de un mostrar enactivo del modo de uso de la cuchara sin que nada nos obligue a suponer que está fingiendo que come. Entre el significante —el gesto que denota el modo de uso del instrumento - y el significado — el modo de uso del instrumento- no hay casi diferencia; y a esto parece reducirse la acción del niño, al reconocimiento de su modo de uso. No tenemos elementos suficientes para suponer que ellos representan objetos o eventos ausentes, ni que haya simulación o ficción alguna.

Podemos interpretar estas actividades de un modo distinto y más simple: como una labor de amalgamar el gesto — que denota el significado aprendido con los otros en relación a un objeto particular- y el objeto. Como una labor semiótica en la que gesto y objeto se amalgaman una y otra vez certificando su enlace. Y a través de la certificación de este enlace el niño se apropia de los objetos de un modo distinto al clásicamente deíctico. Cuando un niño señala y mues- 


\section{4}

tra objetos el acento está puesto en la orientación de la atención hacia el objeto; pero cuando realiza un gesto de reconocimiento el objeto asciende al carácter de algo culturalmente investido que tiene modos específicos de ser usado.

Pero algo realmente extraño ocurre unos pocos meses después: si aproximadamente a los doce meses observamos la presencia de gestos de reconocimiento; a partir de los quince meses, las acciones instrumentales descontextualizadas, abreviadas y rápidas, realizados reiteradamente meses atrás, se hacen con otro objeto o un objeto inadecuado, incluso cuando el objeto adecuado se encuentra en el campo perceptual del niño.

Al 1; 3 (08) S. tiene una cuchara en la mano que ha traído de la cocina; estamos en la sala y S. pasa la cuchara por su cabeza como si fuese un peine. Saco el peine de la bolsa y se lo muestro: S. me mira pero sigue pasando la cuchara por su cabeza. El mismo día usa una cuchara para peinar a sus muñecos. El gesto es breve y pasa rápidamente de un muñeco a otro. El cepillo está a su lado, pero usa la cuchara.

Al 1; 4 (26) coge una goma y peina a su madre, quien le dice que no, que eso no es un peine. S. pasa la goma por una carpeta y mira a la madre que le dice que sí, que es una goma, que así la usa su hermano. S. coge el cepillo, lo deja, coge la goma y peina a su madre.

No hay duda que la niña sabe cómo se usa la goma y así se lo indica a su madre, pero por algún motivo ella quiere usarla de un modo distinto, no convencional. Resulta especialmente llamativo que estas permutaciones de objetos se realicen, a veces, combinando en una misma secuencia la realización del gesto con el objeto adecuado y con el objeto sustituto. Por ejemplo:

Al 1; 4 (26) S está peinando alternativamente a sus muñecos y a su madre. Mira hacia la mesa, suelta el cepillo se dirige hacia allí y coge una cuchara, coge un muñeco, me lo da y lo peina con la cuchara. Luego coge de nuevo el frasco y el cepillo y lo peina como acostumbra, haciendo que pone perfume y luego peinando.

Tanto Habib como Silvita reconocen estos objetos — cucharas, peines, vasos - por su uso y han realizado frecuentemente con ellos gestos de reconocimiento. Esto nos brinda la certeza de que los niños, en las situaciones descritas, no confunden un objeto con otro, no cometen un error de categorización. Más bien parece que han encontrado un nuevo modo de usar los objetos, que no se consigue a través de titubeos o sucesivos intentos, sino de modo directo; hay una intención del niño de usar una cosa como si fuese otra. Intención que se torna particularmente evidente en la última observación cuando S., que está peinando con un cepillo a su madre, deja el cepillo, se dirige hacia la cuchara que está sobre la mesa y peina con ella a su madre.

Si hasta los catorce meses la percepción del objeto despierta en el niño la realización del gesto que denota su modo de uso; en este momento, la relación gestoobjeto parece quebrarse. El gesto se desprende de su objeto y se produce un cambio en la dirección de la actividad del niño: ésta no va del objeto al gesto, como ocurre en los gestos de reconocimiento, sino del significado al gesto. Pero el gesto sólo puede desprenderse de su objeto adhiriéndose a otro objeto también desprendido de su gesto de reconocimiento. Por tal motivo los hemos llamado gestos permutados (Español, 2001a).

En los gestos permutados se perciben dos movimientos: un movimiento libera el gesto de su objeto, otro permite que el gesto se enlace a otro objeto. Por ejemplo, en el caso del gesto permutado de peinarse con una cuchara, parece haber (1) un movimiento en el que el objeto (peine) se separa del gesto (peinar) y éste queda "libre". (2) otro movimiento en el que se suspende el conocimiento 
sobre el uso convencional de un objeto (la cuchara) quedando el objeto "libre" de su gesto de reconocimiento. Gestos y objetos libres pueden así combinarse uno con otro.

En síntesis, si a los doce meses la labor del niño es amalgamar los gestos con sus objetos,
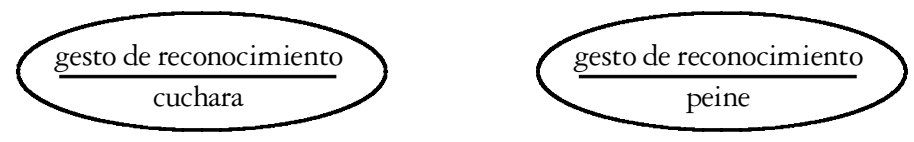

alrededor de los quince meses éstos se separan dando lugar a permutaciones de objeto-gesto.

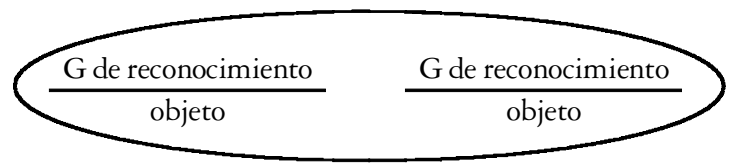

La cuestión es ¿por qué el niño realiza con otro objeto un gesto que hasta ese momento estaba adherido al objeto adecuado?, ¿por qué utiliza un objeto inadecuado cuando podría realizarlo del modo habitual ya que el objeto adecuado, en muchas ocasiones, está a su alcance?, ¿por qué quiebra aquello que fue amalgamado meses atrás? Los gestos de reconocimiento y los gestos permutados se presentan, frecuentemente, combinados en una misma secuencia. Estas combinaciones en las que se juntan y separan, una y otra vez, gesto y objeto, inducen a pensar que el niño juega con la relación misma gesto/objeto. Y este juego prepara el terreno para la construcción de gestos simbólicos: se muestra como el primer paso en el tránsito de gestos presimbólicos, arraigados al objeto, a gestos en los que entre objeto y gesto media una distancia. Es decir, en los gestos deícticos y los gestos de reconocimiento, el objeto se encuentra anclado al gesto; en momentos posteriores del desarrollo, gesto y objeto se separarán al punto de que el primero podrá representar al segundo, y podrá evocarlo en su ausencia. El quiebre que estoy describiendo entre gesto y objeto parece el primer paso hacia la distancia efectiva entre ambos. Un primer paso en el que se manifiestan aún las limitaciones de los gestos presimbólicos: el niño únicamente puede separar el gesto del objeto usando como pivote otro objeto, agarrándose a él. Sirviéndose de otro objeto como pivote, los gestos permutados parecen iniciar el camino que transita de los gestos anclados a sus objetos a los gestos simbólicos.

Pero además, en estas permutaciones también el significado se separa del objeto; y este distanciamien to es también un paso previo necesario para la emergencia de los símbolos. La cualidad específica de los gestos simbólicos (y de los símbolos en general) es que ellos evocan un objeto, que no es ya "el objeto en el mundo" sino el significado que el niño ha ido construyendo con los otros acerca de ese objeto. Es decir, los símbolos aparecen cuando los significados estables pueden ser objetos de referencia. Estas acciones de los niños parecen favorecer el proceso a través del cual el significado empieza a adquirir cierta independencia, abriendo así las puertas a que él mismo —el significado-, ya no el objeto, pueda ser el objeto de referencia de un acto semiótico. 


\section{Ritualización y creación de escenas}

He hablado de los gestos de reconocimiento, que aparecen alrededor de los doce meses, y de los gestos permutados que surgen unos pocos meses después. Querría ahora retornar a los gestos de reconocimiento, y a las acciones descontextualizadas realizadas con objetos adecuados, y describir la evolución que ellos sufren y a la que los gestos permutados parecen ser, en principio, inmunes. A esta evolución le hemos dado un nombre un tanto extenso: "proceso de ritualización en ausencia de asimilación deformante”, para vincularlo y a la vez diferenciarlo de la ritualización piagetiana (Español, 2001a).

Casi a la vez que los niños realizan sus primeros gestos de reconocimiento, en la que ellos mismos son los receptores de la acción, éstos se extienden hacia otros receptores: los adultos, sus muñecos (llevan la cuchara vacía a la boca de sus muñecos o a la de los adultos, acercan el auricular a la oreja de los otros, o llevan un vaso vacío a la boca de la muñeca). La ampliación de los posibles receptores de la acción es el primer paso de un proceso de ritualización que se continúa, a partir de los quince meses, en complejas combinaciones en las que empiezan a configurarse "escenas" que contienen varios instrumentos (cucharas, platos, vasos), varios actores (el niño, el adulto, los muñecos) que pueden ser posibles receptores o agentes de la acción. Estas escenas con contenidos específicos se despliegan en diversos sentidos como lo muestran las siguientes observaciones.

Al 1; 4 (9) S. trae de la cocina un cepillo y un frasco de plástico. Levanta el frasco y lo coloca sobre mi cabeza, luego me peina. Repite dos veces más la secuencia con el frasco y el cepillo. Va de la madre a mi peinándonos; también peina a la muñeca y a su hermana, siempre de la misma manera. En una de esas ocasiones la madre, después de que $\mathrm{S}$. le ha puesto perfume a un muñeco, se acerca e inspira exageradamente “iqué bien huele!”- dice. La niña incorpora la inspiración exagerada a la secuencia poner perfume, peinar.

Al 1; 4 (11) S. acerca la cuchara vacía a mi boca, pero esta vez abre su boca al mismo tiempo. Rato más tarde vuelve a acercar la cuchara a mi boca, abre la suya y dice "aammm".

Al 1; $4(13) \mathrm{H}$. me acerca la tetera, mientras tiene el vaso en la mano, yo hago que sirvo haciendo "shbhb". H. lleva su boca el vaso vacío. Le doy la tetera, pero me la devuelve. Vuelvo a servir haciendo "shbhb". H. ríe, le gusta el ruido. Repetimos la escena varias veces. Luego $\mathrm{H}$. toma la tetera, la inclina sobre el vaso, la sostiene así un rato mientras dice "shhhb". Se lleva el vaso vacío a la boca, luego lo acerca a la mía y yo hago que tomo.

Cuando $\mathrm{H}$ tiene $1 ; 5(10)$ toma la tetera de plástico e imita el movimiento de servir en un vaso, mientras dice "shbhb", luego toma una cuchara, revuelve en el plato y la acerca a mi boca, yo la abro, iqué rico! — digo. H. toma un plato, pasa la cuchara y se la lleva cerca de su boca, sin ponerla dentro, aunque la abre de un modo exagerado.

Resultaría extraño ver a un niño de más de dieciseis meses solamente llevándose a la boca una cuchara o un vaso vacío, ellos suelen realizar actividades más complejas en las que:

1. Se amplían los receptores de la acción

2. El niño asume más de un rol: rotan los actores en su calidad de agentes o receptores.

3. Combinan el uso descontextualizado de varios instrumentos

4. Secuencian alguna parte de la acción: ciertos pasos se establecen como previos y son seguidos reiteradamente de otros, siempre de igual manera

5. Abrevian o exageran la acción 
6. Modifican el tiempo de duración de alguna parte de la acción

7. Incorporan elementos o sonidos nuevos

Mediante el proceso de ritualización, que en cada ocasión puede implicar uno o más de los recursos señalados, lo que eran gestos de reconocimiento o imitaciones globales se transforman en secuencias complejas que semejan escenas. Ellas están lejos de ser todos indiferenciados, tienen elementos constitutivos que se diferencian claramente unos de otros, que se transforman y se combinan entre sí. A la par que se complejizan, las escenas tienden a estilizarse en el sentido que adquieren una forma propia, un modo particular de ejecutarse, abreviándose unas partes, exagerándose otras, secuenciándose algunos segmentos y combinándose de un modo reiterado. Al realizarse una y otra vez se resaltan las partes constitutivas de diversas acciones instrumentales; se pone así al alcance del niño un conocimiento cada vez más preciso de los componentes de la acción instrumental tornando entonces posible la selección de un fragmento de la acción. Nótese que en el proceso descrito los objetos, aunque de modo deformado, son usados del modo en que convencionalmente se usan, en él no se manifiesta asimilación deformante alguna, es decir no hay todavía sustitución de un objeto por otro (los gestos permutados, en los que puede verse un inicio de sustitución, no forman en este momento partes de las escenas construidas por el niño). Este proceso de ritualización, entretejido con la capacidad y flexibilidad adquirida a lo largo de los meses previos de usar diferentes recursos comunicativos - conductas ostensivas puras, gestos deícticos, imitaciones globales-, permite la creación de los primeros símbolos enactivos. Por ejemplo:

Cuando H. tiene 1; 4 (13) estamos jugando a hacer que bebemos de vasos vacíos, a veces yo sirvo de la tetera, otras es $H$. quien sirve, y lo hace siempre de igual modo: coge la tetera con una mano y la inclina sobre el vaso, manteniendo cierta distancia entre ellos. Luego bebe del vaso o lo acerca a mi boca. En un momento $H$. rechaza el vaso vacío que le doy; hago que sirvo de la tetera y se lo vuelvo a dar; vuelve a rechazarlo alejándolo de sí con la mano; luego coge la tetera, la apoya en el vaso, colocándola casi en la misma línea, me mira y se queda esperando. ¿Quieres agua? — le pregunto- mientras me levanto para llenar un vaso; se lo doy y $\mathrm{H}$. bebe.

H. pide agua, y lo hace de un modo idiosincrático sobre la base de la selección de una parte de la acción instrumental: elige de toda la secuencia que hace a la "escena de beber" el colocar la tetera sobre el vaso y al hacerlo establece contacto ocular. Pero, además, "deforma” su imitación al suspenderla. H. exagera el movimiento de inclinación de la tetera hasta ponerla encima del vaso, deteniendo ahí su acción y mirándome. El corte de su acción, el dejar la tetera apoyada en el vaso dejando en suspenso su continuación, deforma su imitación. Es ese corte lo que lo distancia y diferencia del juego funcional en el que estamos inmersos, lo que lo transforma en un símbolo enactivo mediante el cual pide un objeto ausente: el agua. El niño ha inventado un símbolo y le ha dado resultado, ha sido interpretado.

Al 1; $6(04) \mathrm{H}$. está descalzo y pone un pie encima de la mesa. "No, el pie en la mesa no" - le dice la madre. H. la mira, pero no baja el pie sino que acerca su mano y, con el índice y el pulgar juntos, toca su uña y vuelve a mirarla. Baja ese pie sube el otro y hace lo mismo mientras mira a la madre. Eso es nuevo, ¿qué haces? -le pregunta la madre. H. sube de nuevo el otro pie y repite el gesto mirando a su madre. “ $i A h$ ! te estás cortando las uñas como mamá y papá — exclama sonriendo”. H ríe. Va a sillón se sube y repite el gesto mirándonos a la madre y a mí alternativamente. No se puede tener intimidad contigo — comenta con humor la madre. 


\section{8}

En esta ocasión H. busca compartir una situación que en ese momento él evoca; una situación que implica un instrumento y el modo en que sus padres lo utilizan; H. cuenta algo que sabe acerca del mundo, lo plasma enactivamente mediante la imitación, breve, de una de sus partes.

Entre los diversos símbolos creados por Habib y Silvita quiero resaltar especialmente algunos que recuerdan el primer símbolo enactivo descrito por Ángel Rivière (1984) en su artículo Acción e interacción en el origen del símbolo en el niño.

Cuando H. tiene 1; 6 (11) estamos sentados en el suelo de la sala de su casa. H. coge un mechero, me lo da y, mientras está en mi mano, toca la piedra como si quisiera prenderlo. Lo dejo en el suelo. H. agarra el encendedor y lo apoya, dos veces, en un plato, en línea oblicua, en acción solitaria . Cojo de la repisa una vela grande dentro de un recipiente de metal y la dejo en el suelo. H. la toma, me la da, la señala y sopla la vela apagada mientras me mira. Enciendo la vela, H. sopla y la apaga.

El niño, al principio, parece querer prender el mechero. Luego realiza un gesto de reconocimiento, acerca el mechero al plato en línea oblicua, como si dijera "esto se usa así ". En cuanto traigo la vela su acción se orienta hacia mí, me pide que prenda la vela. Su forma de pedir es original: señala y sopla la vela apagada, buscando enseguida el contacto ocular. La llama ha sido simbólicamente evocada mediante un soplar no funcional —el soplido no apaga nada- Como dice Rivière (1984, 1990), las acciones con las que se constituyen los símbolos enactivos son simbólicas precisamente porque no son funcionales; son acciones simbólicas en tanto se desgajan de su funcionalidad de primer orden para adquirir una funcionalidad nueva: la de ser capaces de representar algo. Es decir, con ciertas acciones inadecuadas desde un punto de vista funcional, o mejor dicho, con acciones desgajadas de su función de primer orden, el niño representa ciertos significados.

Cuando H. tiene 1; $6(17)$ prendo una vela pequeña y la muevo haciendo ochos grandes frente a él. La apago. H. toma el mechero y me lo da; prendo la vela. $H$. no la apaga como otras veces sino que mueve su mano, como si hiciera ochos y me mira. Repito el movimiento con la vela prendida; luego la apago. $\mathrm{H}$. vuelve a darme el mechero y la vela. Yo cruzo las piernas y giro mi cabeza para otro lado, haciendo que no le presto atención; H. se acerca y busca mi cara, nos miramos y reímos; y así seguimos un rato. De pronto, $H$. coge la vela apagada que tenía en mis manos, la sopla y la mueve hacia los costados. La cojo y la prendo. Pero H. se aleja, ha cogido otra vela y repite el movimiento sólo para él.

La flexibilidad de los recursos comunicativos de $\mathrm{H}$. es asombrosa; primero me indica que quiere que prenda la vela acercándome el mechero; cuando desea que mueva la vela que está prendida, imita en seguida el movimiento que quiere que haga. Y luego, cuando la vela está apagada construye un símbolo complejo en el que amalgama la acción de soplar disfuncional con la imitación del movimiento. Yo supongo que quiere que se repita el movimiento con la vela encendida, pero cuando prendo la vela, $\mathrm{H}$. se va; tal vez su acción era una declaración y no una petición. Llamativamente, el niño se aleja solo con una vela apagada en su mano repitiendo el movimiento: ¿se trata de un símbolo enactivo autodirigido? También en la siguiente observación parece combinarse el uso de símbolos dirigidos a otros y autodirigidos.

Al 1; 6 (28) S. abre una caja de madera, coge un mechero y sopla dirigiendo su mirada sólo al mechero; luego gira y se dirige hacia la madre con el mechero en la mano y el brazo a medio extender hacia adelante, cuando está cerca de ella, sopla dos veces y se lo da. La madre lo prende varias veces. "Basta" —le dice 
luego- mientras tiene el mechero en su mano; la niña lo coge y sopla nuevamente el mechero apagado. "No — vuelve a decirle la madre.

Tal vez por mi insistencia en elicitarlo, tal vez porque las llamas atraen poderosamente la atención de los niños, pero lo cierto es que el símbolo se ha establecido en el repertorio gestual de ambos niños y hemos pasado largo rato prendiendo y soplando velas. Incluiré una última observación.

Cuando H. tiene 1; 7 (25) toma una vela pequeña, sopla y me mira, busco el encendedor y la prendo. Como $\mathrm{H}$. no la apaga, lo hago yo y la dejo en el suelo. $\mathrm{H}$. coge la vela apagada, sopla y dice no con la cabeza mientras me mira y ríe. Río con él.

En esta ocasión Habib reitera su símbolo enactivo y yo enciendo la vela. Pero parece que no es eso lo que el niño quiere. Su último símbolo, compuesto por el soplar y negar con la cabeza, parece decirme "oye, que esto es un declarativo, no la prendas”. Ambos nos reímos, no sé bien porqué lo hace el niño, yo sé que interpreté su símbolo como una broma que denotaba un nivel de recursividad, de opinar sobre sus propios gestos y las interpretaciones que ellos despertaban en mí, que me asombró.

Además de estos símbolos vinculados con acciones instrumentales hemos observado algunos otros que se relacionan con secuencias rítmicas. Ellos también implican imitación, selección de un fragmento y suspensión de un todo mayor; es decir son también genuinos símbolos, metonimias enactivas pero creadas sobre la base de secuencias rítmicas. Secuencias que son, al igual que las acciones instrumentales, un lugar privilegiado en el ejercicio de segmentar y reconocer partes de un todo (Español, 2001a). Por ejemplo:

Al 1; 7 (25) estamos en la sala de su casa, hay un vídeo puesto. $H$. se levanta, me mira y mueve sus brazos y manos imitando una parte de los movimientos con los que se baila el flamenco. — ¿Quieres el vídeo de Joaquín Cortés?”- le pregunto mientras me levanto para buscarlo.

Una de las actividades preferidas de $\mathrm{H}$. es ver este vídeo. En numerosos ocasiones ha bailado imitando los movimientos de piernas y brazos, variando las velocidades, pasando una mano por el brazo opuesto, como ve que hacen los bailarines, girando cada tanto la cabeza, dando vuelta en círculos y zapateando en distintas direcciones. Muchas veces el niño ha pedido que se ponga el vídeo, acercándose al equipo, permaneciendo a su lado, o señalándolo y mirando al adulto. Pero en ésta ocasión $H$. ha construido un símbolo idiosincrático seleccionando una de las tantas partes que compone la "escena de bailar flamenco" e imitándola mientras me mira.

Los niños producen muchos gestos simbólicos que no son símbolos enactivos. De hecho, el que lo sean o no depende del modo en que se han construido. Si ellos son aprendidos por imitación y el enlace entre significante y significado es, desde la perspectiva del niño, arbitrario, no nos encontramos frente a símbolos enactivos. Pero ahí donde se observen formas claras, precisas, breves, originales y "algo extrañas", ya sea por su disfuncionalidad (como soplar una vela apagada) o por su apariencia deformada debido a la suspensión (como poner una tetera sobre un vaso y dejarla así un rato, o llevarse el índice y pulgar hacia la uña del pie sin tener nada en la mano) es posible sospechar la presencia de un símbolo enactivo; sólo observando su contexto de producción podrá determinarse si realmente se trata de una creación semiótica, si es un modo libre, no copiado, de producción de significado. De un significado que además se encuentra cargado de las experiencias vitales del niño; sus símbolos no sólo remiten a objetos o eventos ausentes sino a su modo particular de concebirlos. 
Un gesto deíctico simplemente orienta nuestra atención hacia algo presente en el entorno inmediato, un símbolo enactivo nos muestra cómo concibe el niño los objetos. No muestra sólo una concepción general del modo de uso cultural y convencional de un objeto, como los gestos de reconocimiento (que las cucharas se llevan a la boca o que los peines se pasan por el pelo); sino que ellos transportan modos particulares de concebirlos que se forjan en situaciones que van conformando un significado cultural pero particularizado de un modo único en cada uno de ellos. Como indica Hobson (1993), los símbolos son vehículos de la concepción de los objetos. Los mecheros no son objetos para prender cigarrillos, modo como por ejemplo los concibo yo, sino objetos que tienen algo llamativo y bello: llamas. Los símbolos nos hablan de las experiencias cotidianas de los niños, algunas tan íntimas como el modo particular que tienen sus padres de cortarse las uñas. Cuando H. era más pequeño hacía saber al otro que deseaba ver el vídeo de Cortés señalándolo; a sus diecinueve meses, frente a ese mismo deseo, construye un símbolo idiosincrático. La diferencia entre ambos modos gestuales pone de relieve el carácter genérico de los gestos deícticos, que no varían sustancialmente en función del referente, en contraste con los símbolos enactivos que especifican en su propia forma la naturaleza del referente. Lo que el niño evoca a través de su símbolo es un modo de relación con el objeto; el símbolo está, no por el objeto, sino por una forma de relación concreta con el objeto que se especifica a través de él. Los símbolos enactivos son formas precisas, sintéticas y originales de significar que, justamente por eso, por ser originales, permiten ver el modo en que el niño concibe los significados referidos. Y en un movimiento en espiral, el símbolo modifica a su vez el significado. Como dijo Ángel Rivière (1990) la construcción del significante, mediante la deformación de una acción, modifica, a su vez, el significado original. Los significantes construidos por el niño no sólo remiten a sus significados o representaciones mentales, sino que los condicionan interpretan y desbordan.

\section{HACIA LA CREACIÓN DE FICCIÓN}

He hablado de los gestos de reconocimiento, que surgen alrededor de los doce meses, y he descrito dos procesos o transformaciones que ellos sufren: (1) los gestos permutados, que surgen cerca de los quince meses, en los que se quiebra la relación gesto-objeto, y (2) el proceso de ritualización, que se despliega también a partir de los quince meses, en el que se desglosan las partes constitutivas de las acciones instrumentales. El análisis que realicé estuvo únicamente orientado a mostrar cómo ambos tornan posible unos meses después, alrededor de los dieciocho meses, la creación de símbolos enactivos. Retornaré ahora a ellos pero desde otra perspectiva.

\section{Conformación de escenas y simulación}

Al hablar de los gestos de reconocimento señalé que no teníamos elementos suficientes para suponer que el niño, al realizarlos, estuviera simulando o haciendo "como que"; pero a medida que el proceso de ritualización avanza es posible ver cómo se gesta una incipiente capacidad de simulación. Si se comparan las observaciones descritas en el punto anterior correspondientes a los doce meses y a los meses posteriores podrá percibirse la creación paulatina de lo que Rivière (1997) denomina un "marco" que denota un terreno o cuadro posible de ser 
habitado de "modo simulado". La construcción de pequeñas secuencias estereotipadas y reiteradamente actuadas por el niño abre las puertas a la constitución de un escenario que puede habitar de un "modo simulado" manteniendo en la conciencia dos planos de realidad: uno en el que las acciones tienen sus efectos habituales, otro en el que no. La constitución de este escenario no es un resultado de la actividad solitaria del niño, los adultos co-participan en el proceso de ritualización construyendo con él las escenas a que éste da lugar. Frecuentemente están involucrados como intérpretes, receptores y agentes de las acciones que en ellas se despliegan, muchas veces introducen elementos nuevos de los cuales los niños inmediatamente se apropian. Les muestran, así, una cierta actitud de simulación. Si ellos no completaran la acción del niño, si no se involucraran, por ejemplo, abriendo la boca cuando se les acerca una cuchara vacía, sería poco probable que el proceso de ritualización, que empieza justamente con la extensión de los receptores de la acción, se continuase hasta dar lugar al "modo simulado". Es todo el complejo de conocimientos de usos convencionales de objetos, de actitudes psicológicas, de interacciones lúdicas lo que parece subyacer a la capacidad de vincularse con la realidad de un modo novedoso: el modo simulado.

Un modo en el que los niños simulan que ellos u otros realizan ciertas acciones básicas de la vida cotidiana (como beber o comer) usando los objetos de forma descontextualizada, deformada, pero respetando su uso convencional. Por tal motivo hablo de simulación y no de ficción. La ficción que va unida con la presencia de sustitución de objetos, con el ver un cosa y actuar como si fuera otra, aparece en momentos posteriores. Sin embargo, en esta tempranas simulaciones es posible ver una alusión simbólica a eventos de la vida cotidiana. Pese a que las escenas no parecen compuestas por símbolos, pareciera que de ellas brotara un efecto simbólico que no puede adscribirse a ningún sitio específico sino que emerge del conjunto. Por ejemplo, el líquido está de alguna manera evocado por el conjunto "servir con una tetera en un vaso, mientras se demora el movimiento y se hace algún ruido, llevando después el vaso a la boca”. Pero no se trata todavía de que un objeto represente a otro, no se realiza sustitución alguna, sino que es el conjunto el que evoca objetos o eventos no presentes ${ }^{1}$.

\section{Sustitución y ficción}

La creación de ficción es el tercer nivel de la teoría de semiosis por suspensión. La explicación de ficción por suspensión es una propuesta alternativa a la teoría de Leslie (Leslie, 1987, 1988, 1994; Leslie y Roth, 1993) en la que se destaca un cierto isomorfismo entre el juego de ficción y la propiedad lógica de intensionalidad que tienen los enunciados con verbos de creencia y en la que se explica tal isomorfismo apelando a la existencia de metarrepresentaciones y a la operación con proposiciones y actitudes proposicionales. La teoría de semiosis brinda una explicación más simple. En ella se sostiene que una vez que se han constituido representaciones primarias estables acerca del mundo éstas se tornan factibles de quedar en suspenso dando lugar al juego de ficción. Al quedar en suspenso las representaciones primarias, las affordances o las propiedades mismas de los objetos se anticipa la propiedad lógica de intensionalidad sin que sea necesario postular que el niño opera con proposiciones y actitudes proposicionales. No pretendo exponer aquí esta hipótesis (para su desarrollo ver Rivière, 1997; para un comentario acerca de ella ver Español, 2001b). Mi objetivo es más medido, querría mostrar cómo se produce el tránsito al tercer nivel de suspensión poniendo el acento, como hasta ahora, en los aspectos morfológicos. La hipótesis que intenta- 
ré desarrollar es que la capacidad de dejar en suspenso las representaciones de los objetos se encuentra mediatizada por la construcción de escenas en las que el niño se sitúa de un modo simulado y por la inclusión de los gestos permutados en ellas. Ello me llevará a plantear la cuestión en términos de sustitución de un objeto por otro. La dupla ficción-sustitución es un foco de polémica en psicología del desarrollo. Para algunos la sustitución de objetos no es un rasgo esencial del juego de ficción (Bates, 1979); para otros es casi su espina dorsal (Piaget, 1946). Algunos insisten en que puede haber apariencia de sustitución sin que ésta efectivamente ocurra y que la sustitución simbólica únicamente puede realizarse a través del lenguaje (Vygotski, 1933, 1934; Elkonin, 1978). Otros pregonan que sustitución no es equivalente o sinónimo de simbolización (Perner, 1991, 1993; Lillard, 1993). A continuación defenderé la idea de que la sustitución es una cuestión de grado. Intentaré mostrar que existen diferentes tipos de sustituciones siendo algunas de ellas sustituciones simbólicas y otras no. Haré uso de la gramática de casos de Fillmore (1968) para resaltar las diferencias entre unas y otras.

La primera impresión de que se sustituye un objeto por otro la encontramos alrededor de los quince meses cuando emergen los gestos permutados. Ellos parecen iniciar un nuevo modo de tratar con los objetos en los que la sustitución de uno por otro tiene por primera vez lugar. Pero si se recuerdan las observaciones descritas casi al inicio de este trabajo, se verá que ellos presentan dos claras restricciones: (1) la sustitución ocurre siempre en un mismo caso: el caso instrumento, (2) el modo de sustitución es particular: sólo puede sustituirse un objeto con un uso convencional conocido por otro objeto de iguales características. Por otro lado ellos suelen realizarse aunque el objeto adecuado se encuentre en el campo perceptual del niño, y muchas veces se combinan con el gesto realizado con el objeto adecuado. Ahora bien, ¿qué relación se establece entre el instrumento actual y el original?, ¿representa el instrumento sustituto al instrumento sustituido? Tanto las restricciones señaladas como su modo de presentación inducen a pensar que la relación entre el objeto sustituto y el objeto sustituido no es de representación, el objeto sustituto no "está por" o "no representa a" el objeto sustituido; simplemente se usa uno como se usa el otro. El niño sólo puede ver un objeto convencional y usarlo como si fuese otro objeto convencional. No es ésta una gran libertad, es cierto, pero parece el germen de la creación de ficción en las que se puede libremente utilizar una cosa cualquiera para significar otra.

El tránsito desde este primer modo de sustitución a la genuina sustitución simbólica es paulatino y se encuentra estrechamente unido al desarrollo y complejización de las escenas antes mencionadas. Pese a que los gestos permutados aparecen en momentos tempranos del desarrollo (y se presentan a veces en breves combinaciones) no se incluyen en las escenas hasta aproximadamente los dieciocho meses. Da la impresión de que el niño necesita transitar con cierta maestría en lo que podemos llamar vector simulado-real para que los gestos permutados, en los que el niño empieza a actuar independientemente de los que ve, puedan ser incorporados. Poco a poco, ellos empiezan a integrarse en la escenas, al principio de un modo breve en el que, a veces, se cambia "el tema" rápidamente volviendo a usar el objeto de modo adecuado.

Al $1 ; 6(17) \mathrm{H}$. sirve con la tetera en una vela grande la lleva a su boca y chasquea la lengua contra el paladar. Sirve nuevamente de la tetera haciendo "shbhb", hace que bebe de la vela y nuevamente chasquea la lengua. 


\section{3}

Al 1; 7 (11) S. está peinando a su madre con un peine. Revuelve con el peine en la tetera y le da de comer reiteradas veces a la madre; extiende el peine hacia mí que estoy a la distancia, mientras ambas abrimos exageradamente la boca.

$\mathrm{Al} 1 ; 7(25) \mathrm{H}$. pone la cuchara en la vela grande, revuelve y se lleva la cuchara a la boca mientras dice "ammm", luego revuelve en un plato. Coge un peine revuelve con él y se lo lleva a la boca, inmediatamente se peina, lo deja y coge unos cubos.

Durante los meses siguientes las escenas se extienden y aparece un hilo conductor que permite enlazarlas: generalmente el muñeco ${ }^{2}$. El niño permanece largo rato en el modo simulado, sin confundirlo con el modo real, y las escenas comienzan a parecer pequeñas narraciones en acción. Ellas recuerdan lo que Bruner (1990) llamó la facilidad o predisposición a organizar la experiencia de forma narrativa. $\mathrm{O}$, en otras palabras, ponen de manifiesto la hipótesis de que la estructura narrativa se encuentra presente en la interacción social antes de adquirir su expresión lingüística y provee de una cierta predisposición prelingüística para el significado. El impulso a construir narraciones parece motivar la creación de escenas en las que se simulan o representan sucesos de la vida cotidiana y en las que la agencialidad y el orden secuencial (dos de los cuatro constituyentes gramaticales fundamentales de las narraciones) son jugados enactivamente, de modo insistente y variado. Y en este despliegue la simulación, vía la sustitución, empieza a adquirir ese rasgo de deformación típico de la ficción. Es decir, es en el seno de estas "casi” narraciones en donde la sustitución simbólica, la ficción, aparece por primera vez

Cuando S. tiene 1; 10 (0) estamos en el jardín con vasos, platos, cuchara y un balde con arena y agua. S. ha llenado un vaso con arena, saca un poco con la cuchara y la pone en un plato; coge el plato y sopla. ¿Está caliente? - le pregunto. "Sî" — responde S. — y le da de comer a la muñeca. Al rato, agrega palitos y césped a la arena. S. se divierte revolviendo y agregando más palitos, luego le da de comer a la muñeca.

En esta situación la arena, el césped y los palitos parecen sustituir a la comida. Sin embargo, aún podría decirse que no son éstas verdaderas sustituciones simbólicas sino que es la escena en conjunto la responsable de generar una ilusión de sustitución. En la siguiente escena tal objeción desaparece y no parece haber duda que estamos frente a una sustitución simbólica de objetos.

Al 1; 10 (19) S. está peinando a la muñeca y se sale uno de los trozos de lana negra que hace de pelo; S. lo coge — dice_— "pelo", se levanta y camina hacia la mesa; pone el pelo en un vaso y vuelve con él hacia la muñeca; coge un peine, pero lo deja, camina hacia la mesa y trae una cuchara. Saca el pelo del vaso, lo pone en la cuchara, camina despacio con el pelo en la cuchara, se acerca a la muñeca y, con cuidado, lleva el pelo a su boca.

En este caso no es el despliegue de la escena en conjunto lo que hace pensar en una sustitución de objetos, sino al revés. La niña sustituye el pelo por la comida en cuanto se desprende de la cabeza de la muñeca, y es la representación (comida) de una representación (pelo) la que guía la constitución de la escena. Ella despierta una planificación de la acción, incita a seguir ciertos pasos: la niña pone el pelo en un vaso, luego busca un objeto con el que darle la comida a su muñeca, coge el peine y lo deja probablemente porque no le sirve, busca la cuchara y entonces vuelve despacio a darle la comida a su muñeca. Lejos estamos de los momentos en que los objetos despertaban gestos de reconocimiento de objetos, e incluso de las primeras sustituciones presentes en los gestos permutados. Ya no se trata de que un objeto es usado como si fuese otro, sino que un objeto representa a otro objeto ausente. La sustitución no recae en el caso instrumento sino 


\section{4}

FIGURA 1

Pasos hacia la constitución de símbolos enactivos y la creación de ficción ${ }^{3}$

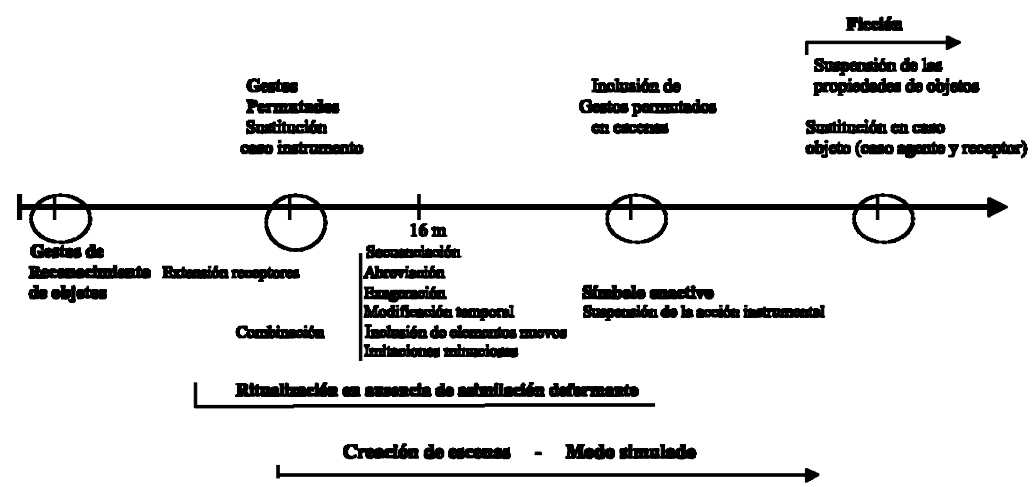

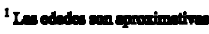

en el caso objeto. Este modo de sustitución simbólica se continúa con otros en la que la sustitución recae en los casos agente y receptor, pero este artículo se ha tornado demasiado extenso para que pueda referirme a ellos (ver Español, 2001a).

Nos encontramos en el tercer nivel de la teoría de semiosis por suspensión, aquél en el que se pueden dejar en suspenso las representaciones de los objetos generando juego de ficción. Lo que la define como tal es la operación de la suspensión sobre una nueva fuente, las representaciones primarias o las propiedades, las affordances, de los objetos que permite la sustitución simbólica de un objeto por otro. La figura 1 muestra el proceso de constitución de los símbolos enactivos y los pasos hacia la creación de ficción. En ella se sintetiza la idea de continuo que he intentado plasmar a lo largo del texto.

Un último comentario: si los productos de los dos primeros niveles de suspensión, los gestos deícticos y los símbolos enactivos, pueden describirse como metonimias, metonimias enactivas y metonimias instrumentales respectivamente; en el producto del tercer nivel parece anticiparse un movimiento metafórico que lo vincula con el cuarto y último nivel de suspensión (la comprensión metafórica). En el juego de ficción se sustituye una totalidad por otra en un contexto que semeja un marco narrativo. Probablemente sea esta anticipación de un movimiento metafórico en una trama casi narrativa lo que tiñe al juego de ficción de una cualidad especial, lo torna inasible, haciéndonos siempre sentir que lo esencial se nos ha escapado; tal vez sea éste también el motivo por el que frecuentemente se lo ha vinculado con la expresión artística, inasible también, siempre un poco más allá de nuestra comprensión.

\section{Notas}

Nótese la diferencia entre las escenas de las cuales brota un efecto simbólico y los símbolos enactivos que son genuinas formaciones simbólicas. Los símbolos enactivos nos hablan de formas simbólicas previas a la capacidad de simbolización vía la ficción en las que no hay aún sustitución de un objeto por otro.

Para un análisis detallado del uso convencional y exageradamen te peculiar de los muñecos a través del cual los padres realizan con sus hijos un proceso de andamiaje hacia la ficción, ver Español, 2001a.

3 Las edades son aproximativas 


\section{Referencias}

BAtEs, E. (1976). Language and Context: The acquisition of pragmatics. Nueva York: Academic Press.

BATES, E. (Ed.) (1979). The emergence of symbols: cognition and communication in infancy. Nueva York: Academic Press.

Bretherton, Y., Mc New, S. y Beeghly-Smith, M. (1981). Early person knowledge as expressed in gestural and verbal communication: When do infants acquire a "theory of mind". Developmental Psychology, 18, 906-921.

Bruner, J. S. (1991). Actos de significado. Madrid: Alianza.

Butter worth, G. (1998). Origins of social visual attention in infancy. Monographs of the Society for Research in Child Development, 225, 63 (4), 144-166.

Camaioni, L., Perucchini, P., Murator, F. y Milone, M. (1997). Brief report: A longitudinal examination of the communicative gestures deficit in young children with Autism. Journal of Autism and Developmental Disorders, 27 (6) 715-725.

Delgado, B., Gómez, J. C. y SARriá, E. (1999). Non communicative pointing in preverbal children. Poster presentado en IXth European Conference on Developmental Psychology, Isla de Spetses.

Elkonin, D. (1985). Psicología del juego. Madrid: Visor.

ESPAÑOL, S. (2001a). Un estudio de semiosis evolutiva: los primeros gestos comunicativos y simbólicos. Tesis Doctoral. Universidad Autónoma de Madrid.

EsPAÑOL, S. (2001b). Un modo particular de concebir el símbolo y la ficción. En R. Rosas (Ed.), La Mente Re-considerada: En homenaje a Ángel Rivière. Santiago de Chile: Psykhé Ediciones.

Español, S. (En prensa). Aproximación a la teoría de semiosis por suspensión de Ángel Rivière. En J. L. Linaza (Ed.), Cultura y Educación en el Desarrollo Humano. Homenaje a Ángel Rivière, La Rábida: Universidad Internacional de Andalucía.

Español, S. y Rivière, A. (2000). Gestos comunicativos y contextos interpersonales: un estudio con niños de 10 a 16 meses. Estudios de Psicología, 65-66, 225-245.

Fillmore, C. J. (1968). The case for case. En E. Bach y R. T. Harms (Eds.), Universals in linguistic theory. Nueva York: Holt, Rinehart and Winston.

Franco, F. y Butterworth, G. (1996). Pointing and social awareness: declaring and requesting in the second year. Journal of Child Language, 23, 307-336.

GÓmEZ, J. C., SARriÁ, E. y TAMARIT, J. (1993). The comparative study of early communication and theories of mind: ontogeny, phylogeny and pathology. En S. Baron-Cohen, H. Tager-Flusberg y D. Cohen (Eds.), Understanding other minds: perspective from autism (pp.397-426). Oxford: Oxford University Press.

GómeZ, J. C. y NuÑez, M. (1998). La mente social y la mente física: desarrollo y dominios de conocimiento. Infancia y Aprendizaje, 84, 5-32.

GoOdhart, F. y BArON-Cohen, S. (1998). How many ways can the point be made? Evidence from children with and without autism. First Language, 13, 225-233.

Hobson, R. P. (1993). El autismo y el desarrollo de la mente. Madrid: Alianza.

Iverson, M., CAPIRCI, O. y CASELLI, M. (1994). From communication to language in two modalities. Cognitive Development, 9, 23-43.

Leslie, A. M. (1987). Pretense and Representation: The origin of "Theory of Mind". Psychological Review, $94,4,412-426$.

LesLIE, A. M. (1988). Some implications of pretense for mechanisms underlying the child's theory of mind. En W. Astington, P. L. Harris y D. Olson (Eds.), Developing theories of mind (pp. 19-46). Cambridge: Cambridge University Press.

LesLie, A. y Roth, D. (1993). What autism teaches us about metarrepresen tation. En S. Baron-Cohen, H. Tager-flusberg y D. Cohen (Eds.), Understanding other minds: perspectives from autism (pp. 83-11). Oxford: Oxford University Press.

LEsLIE, A. M. (1994). Pretending and believing: issues in the theory of ToMM. Cognition, 50, 211-238.

Lillard, A. (1993). Pretend play Skills and the child's theory of mind. Child Development, 64, 348-371.

MASUR, E. F. (1990). Gestural development, dual direction signaling, and the transition to words. En V. Volterra y C. J. Erting (Eds.), From gesture to language in hearing and deaf children (pp. 18-30). Nueva York: Springer-Verlag.

Perner, J. (1991/1994). Comprender la mente representacional. Barcelona: Paidós.

Perner, J. (1993). The theory of mind deficit in autism: rethinking the metarepresentation theory. En S. Baron-Cohen, H. Tager-flusberg y D. Cohen (Eds.), Understanding other minds: perspectives from autism (pp. 112-137). Oxford: Oxford University Press.

Perucchini, P. y Camaioni, L. (1993). When intentional communication emerges? Developmental dissociation s between declarative and imperative functions of the pointing gesture. Poster presentado en The BPS Annual Conference, Developmental Section, Birmingham.

Phillips, W., Gómez, J. C., Baron-Cohen, S., LaA, V. y Rivière, A. (1995). Treating people as objects, agents, or "subjects": How young children with and without autism make requests. Journal of Child Psychology and Psychiatry and Allied Disciplines, 36 (8), 1383-1398.

PIAGET, J. (1946/1977). La formación del símbolo en el niño. Imitación, juego y sueño. Imagen y representación. México: Fondo de Cultura Económica. 
Rivière, A (1984). Acción e interacción en el origen del símbolo. En J. Palacios, A. Marchesi y M. Carretero (Comps.), Psicología Evolutiva. Vol 2. Desarrollo cognitivo y social del niño (pp. 145-174). Madrid: Alianza.

Rivière, A. (1990). Origen y desarrollo de la función simbólica en el niño. En J. Palacios, A. Marchesi y C. Coll (Comps.), Desarrollo psicológico y educación (pp. 113-130). Madrid: Alianza.

Rivière, A. (1997). Teoría della mente e metarappresen tazione. En F. Braga Illa. (Ed.), Livelli di rappresentazione (pp. 351-410). Urbino: Quattro venti.

RIvière, A. (1998a). El tratamiento del autismo como trastorno del desarrollo: principios generales. En A. Rivière y J. Martos (Comps.), El tratamiento del autismo. Nuevas Perspectivas (pp. 23-59). Madrid: IMSERSO.

RIVIÈRE, A. (1998b). Tratamiento y definición del espectro autista I: relaciones sociales y comunicación. En A. Rivière y J. Martos (Comps.), El tratamiento del autismo. Nuevas Perspectivas (pp. 61-106). Madrid: IMSERSO.

RIvière, A. (1998c). Tratamiento y definición del espectro autista II: anticipación, flexibilidad y capacidades simbólicas. En A. Rivière y J. Martos (Comps.), El tratamiento del autismo. Nuevas Perspectivas (pp. 107-160). Madrid: IMSERSO.

RiviÈRe, A. y Español, S. (En prensa). La suspensión como mecanismo de creación semiótica. Rivière. A. Compilación de escritos. Vol II. Madrid: Editorial Médica Panamericana.

Rivière, A. y NúÑEZ, M. (1996). La mirada mental. Buenos Aires: Aique.

Rivière, A. y Sotillo, M. (1999). Comunicazione, sospensione e semiosi umana: le origini della pratica e della compresione Enterpersonal i. Ricerche di sociologia e psicologia della comunicazione, 1, 45-76.

Rodriguez Garrido, C. y Moro, CH. (1999). El mágico número tres. Cuando los niños todavía no hablan. Barcelona: Paidós.

Tomasello, M. y Camaioni, L. (1997). A comparison of the gestural communication of apes and human infants. Human Development, 40, 7-24.

Trevarthen, C. (1982). The primary motives for cooperative understanding. En G. Butterworth y P. Light (Eds.), Social Cognition (pp. 77-109). Brighton: Harverster.

VyGOTSKI, L. S. (1933/1979). El juego y su papel en el desarrollo psicológico del niño. En L. S. Vygotski, El desarrollo de los procesos psicológicos superiores. Barcelona: Crítica.

Vygotski, L. S. (1934/1993). Pensamiento y lenguaje. En L. S. Vygotski. Obras escogidas, Tomo II. (pp. 9287). Madrid: Visor. 